\title{
Amoxicillin Utilization Pattern at Governmental Hospitals in Eastern Ethiopia
}

This article was published in the following Dove Press journal:

Infection and Drug Resistance

\author{
Tigist Gashaw (D) \\ Mekonnen Sisay (1D \\ Tewodros Tesfa $\mathbb{D}^{2}$ \\ Yohannes Baye ${ }^{3}$ \\ Firehiwot Amare $\mathbb{D D}^{4}$ \\ 'Department of Pharmacology and \\ Toxicology, School of Pharmacy, College \\ of Health and Medical Sciences, \\ Haramaya University, Harar, Ethiopia; \\ 2 Microbiology Unit, Medical Laboratory \\ Sciences, College of Health and Medical \\ Sciences, Haramaya University, Harar, \\ Ethiopia; ${ }^{3}$ Department of Pediatrics and \\ Neonatal Nursing, School of Nursing and \\ Midwifery, College of Health and Medical \\ Sciences, Haramaya University, Harar, \\ Ethiopia; ${ }^{4}$ Department of Clinical \\ Pharmacy, School of Pharmacy, College of \\ Health and Medical Sciences, Haramaya \\ University, Harar, Ethiopia
}

Introduction: Penicillin is among the highly used antibiotics in most parts of the world, with amoxicillin being the most frequently utilized drug in the category. However, amoxicillin use has been found to deviate from standard treatment guidelines (STGs).

Objective: This study aimed to evaluate amoxicillin utilization patterns based on Ethiopian STGs criteria at four governmental hospitals in Harar town: Hiwot Fana Specialized University Hospital, Jugel Hospital, South East Command III Hospital, and Federal Harar Police Hospital in Eastern Ethiopia in 2016.

Methods: A hospital-based retrospective cross-sectional study was employed using medication records of patients who received amoxicillin in 2016 at four governmental hospitals from May 15 to June 30, 2018. A total of 502 medication records were proportionally allocated based on the ratio of consumption data of each hospital. Simple random sampling was employed to collect the required sample from the sampling frame. The collected data were entered into SPSS version 21 and analyzed using descriptive analysis.

Results: Amoxicillin was used in all age groups, including pregnant and lactating women. The majority $(96.2 \%)$ of patients were from the outpatient departments. Complete blood count was the most laboratory investigation carried out in $24.9 \%$ whereas microbiological culture was not recorded at all. Top three indications include nonspecific upper respiratory tract infections $(15.1 \%)$, pneumonia $(13.5 \%)$ and dental problems $(10.6 \%)$. Non-steroidal anti-inflammatory drugs $(56.2 \%)$ were frequently co-administered agents. An appropriate utilization was made considering indication, dose, frequency and therapy duration in $23.9 \%$ as per the Ethiopian STG. The wrong indication (65.4\%) was the prime reason for inappropriateness, followed by dose (14.6\%) and duration of therapy $(12.2 \%)$.

Conclusion: Amoxicillin utilization was appropriate in less than a quarter of patients. The wrong indication was the main reason for inappropriateness, predisposing to resistance development. Further studies identifying factors related to misuse and sensitivity tests should be the next steps.

Keywords: amoxicillin, utilization, antibiotics, resistance, appropriateness

\section{Introduction}

Antibiotics are the most commonly prescribed drugs worldwide, ${ }^{1-4}$ and the misuse and overuse of antimicrobials is also a common problem worldwide. ${ }^{5-7}$ The inappropriate use of medicines on a wide-scale can have a significant impact on health care costs and the quality of drug therapy and medical care, as well as being a primary contributor to the spread of antimicrobial resistance (AMR) and increased likelihood of adverse drug reactions and encouraging patients' inappropriate reliance on medicines. ${ }^{8-12}$
Correspondence: Tigist Gashaw Department of Pharmacology and Toxicology, School of Pharmacy, College of Health and Medical Sciences, Haramaya University, P.O. Box 235, Harar, Ethiopia Email yekalabel@gmail.com
Infection and Drug Resistance 2021:14 193-203 
The emergence and spread of AMR is a complex problem driven by numerous interconnected factors, ${ }^{5}$ among which prior antimicrobial drug exposure is a vital risk factor for colonization and infection due to a drugresistant pathogen. ${ }^{13}$ Thus, pharmaco-epidemiological studies that evaluate individual exposure to antimicrobial agents are critical. ${ }^{14,15}$ Drug use evaluation (DUE) is a system of ongoing, systematic, criteria-based evaluation of drug uses to ensure that medicines are used appropriately at the individual patient level. It might be drug/ disease-specific and can be structured to assess the actual process of prescribing, dispensing, or administering a medication. The selection of agents for drug utilization research should be based on whether a drug is high-use, high-cost, or high risk. ${ }^{16}$

Penicillin, specifically amoxicillin, is one of the most frequently used categories of antibiotics worldwide. ${ }^{17}$ Amoxicillin is an aminopenicillin that is active against $S$. pyogenes and many strains of $S$. pneumoniae and $H$. influenzae, which are among the common upper respiratory pathogens. The drug is effective in urinary tract infections, sinusitis, otitis media, acute exacerbations of chronic bronchitis, and epiglottitis. It is the most active of the oral $\beta$ lactam antibiotics, effective against both penicillinsensitive and penicillin-resistant $S$. pneumoniae. The addition of a $\beta$-lactamase inhibitor (clavulanate or sulbactam) extends the spectrum to $\beta$ lactamase-producing $H$. influenzae and Enterobacteriaceae. The drug is remarkably nontoxic and most of the serious adverse effects are due to hypersensitivity. ${ }^{18}$

The European Center for Disease Control and Prevention (ECDC) reported that penicillin is the primary antibacterial agents used in wider regions of the European continent. ${ }^{19}$ Report from studies conducted at primary healthcare facilities in Turkey, $^{20}$ Malaysia, ${ }^{21}$ and Ethiopia $^{22}$ showed penicillin accounted for $29.2 \%$, $30.7 \%$, and $51.9 \%$ of the antibiotics prescribed, respectively. Results from primary healthcare facilities in Nigeria $^{23}$ and China ${ }^{24}$ also showed amoxicillin was the most frequently prescribed penicillin, which accounted for $25.4 \%$ and $21.3 \%$ of the prescribed antibiotics. Research from Ethiopia at health centers of Somali Regional State $(33.3 \%),{ }^{25}$ hospital of Southern part $(16.4 \%),{ }^{26}$ Public Primary Healthcare Facilities in Addis Ababa (44.8\%), ${ }^{22}$ and Communities of Bahir Dar City Administration $(64.4 \%)^{27}$ reported amoxicillin utilization prescription was high. Moreover, studies from Uganda, ${ }^{28}$ Indonesia, ${ }^{29}$ Guatemala, $^{30}$ Greece, ${ }^{31,32}$ and Mongolia, ${ }^{33}$ further augment the wider preference of using this drug internationally. ${ }^{34}$ This high prescribing practice might increase the reliability of the community using this drug in self-medication practices. ${ }^{26,35,36}$

Inappropriate antimicrobial use has been quantified in various settings using numerous definitions; however, no established reference standard exists. ${ }^{37}$ Yet, different working groups for chemotherapy have proposed key points for the appropriate use of antibiotics in hospital settings, among which prescribing drugs at their optimal dosing and appropriate duration, adapted to each clinical situation and patient characteristics. ${ }^{38,39}$ There is also a wide consensus that the main factor driving AMR development is the overprescribing of antibiotics ${ }^{40}$ leading to the highest consumption rate of antibiotics. ${ }^{41,42}$ The more the antibiotics are used, particularly when misused, the greater the selective pressure placed on bacteria to acquire resistance genes; hence, the need to limit the use of these medicines to what is necessary and proper is critical. ${ }^{43}$ Moreover, although there are national good prescribing practices and antimicrobial stewardship guidelines, these guidelines have not yet been implemented in practice. There are neither strict local rules, regulations, or antimicrobial use policies in such resource-limited settings, ${ }^{44}$ overlocking antibiotics cost which account for $20-50 \%$ of drug expenditures. ${ }^{45}$ Besides, as there are no studies showing amoxicillin utilization in the selected settings, the current study aimed at evaluating its utilization pattern based on Ethiopian standard treatment guideline (STG) criteria in governmental hospitals at Harar, Eastern Ethiopia.

\section{Materials and Methods}

\section{Study Setting, Design, and Population}

Harar is located $526 \mathrm{~km}$ East of Addis Ababa, the capital city of Ethiopia. ${ }^{46}$ The study was conducted in four governmental hospitals: Hiwot Fana Specialized University Hospital (HFSUH), Jugel Hospital (JH), Federal Harar Police Hospital (FHPH) and South-East command III Hospital (SECIIIH). HFSUH is a tertiary care teaching hospital of Haramaya University and hosts the majority of patient attendees per day from the Harar town and nearby areas. $\mathrm{JH}$ is a regional hospital in Harari regional state. Whereas FHPH and SECIIIH are special government hospitals in which service is primarily delivered to police and military clients and their relatives. A hospital-based retrospective cross-sectional study was employed to 
evaluate amoxicillin utilization patterns using medication records of patients who received the drug in 2016 from May 15 to June 30, 2018.

\section{Sample Size and Sampling Techniques}

Considering all hospitals as a single entity, the sample size was determined by using the single proportion formula $\mathrm{n}=\frac{Z_{\alpha}^{2} P(1-P)}{W^{2}}$

Where: $\mathrm{n}=$ sample size, $\mathrm{Z}=$ confidence level, $\mathrm{P}=$ point estimate of population $\mathrm{W}=$ maximum width tolerable error, $\mathrm{q}=1-\mathrm{p}$ (failure rate) to determine the sample size, the $\mathrm{Z}$ statistics value $=95 \%$ (1.96) with two-sided tolerable error $(\alpha)(\mathrm{W}=5 \%$ or 0.05$)$ and $\mathrm{P}=50 \%(0.5)$. $\mathrm{P}$-value is taken as $50 \%$ for determining adequate sample size since there was no research on the topic selected.

$$
\begin{aligned}
& \text { Hence, } \mathrm{n}=\frac{(1.96)^{2} * 0.5(0.5)}{(0.05)^{2}} \\
& \mathrm{n}=384
\end{aligned}
$$

To increase the representativeness and address few methodological issues the sample size was adjusted to 502 medical cards.

\section{Sampling Techniques}

The aforementioned sample size was proportionally allocated by multiplying a correction factor obtained from the ratio of each hospital's amoxicillin consumption data to that of the total consumption of four hospitals. ${ }^{44}$ Accordingly, medical records containing amoxicillin of 100, 200, 130, and 72 were allotted for HFSUH, JH, $\mathrm{SCIIIH}$, and FHPH, respectively. Simple random sampling was employed to collect the required sample size from the sampling frame prepared from card numbers of prescriptions containing amoxicillin.

\section{Study Variables}

- Drug use evaluation

- Sociodemographic status of the patient

- Indication, dose, frequency, and duration of amoxicillin

- Co-administered medications

- Duration of inpatient hospital stay

\section{Inclusion and Exclusion Criteria}

Medication records of patients who were prescribed amoxicillin were included. Records with missing information from more than one criterion stated for analyzing appropriateness as well as those with incomplete sociodemographic status were excluded.

\section{Data Collection}

A preliminary study was conducted on a small number of medical records at Haramaya Hospital, and minor revisions were made on the structured data abstraction format. Next, data were collected retrospectively using the format from patient medication records.

\section{Data Processing and Analysis}

The collected data were entered into SPSS version 21 (SPSS Inc., Chicago, IL, USA) and checked for cleanness. Descriptive analysis was used to summarize the sociodemographic, clinical, and drug-related factors. The amoxicillin utilization was evaluated using the STG of Ethiopia for General Hospital Criteria (indication for use, dose, frequency, and duration). ${ }^{47}$ Tabular and graphical presentations were used to summarize the findings.

\section{Operational Definition}

Appropriate

Appropriateness was defined based on the four parameters, ie, indication, dose, frequency, and duration of therapy of a specific disease. Therefore, proper utilization was correct if all four parameters were found to be appropriate.

\section{Inappropriate}

Incorrect prescribing in one of the four parameters, ie, indication, dose, frequency, and duration of therapy of a specific disease.

\section{Indeterminate}

When there was missed/unrecorded information in one of the four parameters, ie, indication, dose, frequency, and duration of therapy of a specific disease.

\section{Results}

\section{Sociodemographic Characteristics of Patients}

The study showed that most participants were female $(58.4 \%)$ and in the age group of 25-49 years (50.6\%). Information regarding body mass index (BMI), weight, and height were negligibly recorded in all study areas. Though it seems inconsistent, the drug was also used during pregnancy and lactating mothers (Table 1).

Among patients treated with amoxicillin, nearly half $(\mathrm{n}=259,51.7 \%)$ of the patients were served in the Medical OPD followed by Pediatric OPD ( $\mathrm{n}=89,17.8 \%$ ), Emergency OPD ( $\mathrm{n}=77,15.4 \%)$, and Gynecology and Obstetrics OPD $(\mathrm{n}=58,11.6 \%)$ (Table 2). Complete blood count (CBC) was 
Table I Sociodemographic Characteristics of Patients Who Were on Amoxicillin Therapy in Selected Governmental Hospitals of Eastern Ethiopia in $2016(n=502)$

\begin{tabular}{|l|l|l|l|l|l|l|}
\hline \multicolumn{2}{|l|}{ Sociodemographic Variables } & JH & FHPH & HFSUH & SECIIIH & Overall Freq (\%) \\
\hline Age (y) & $1-9$ & 34 & 17 & 37 & 12 & $100(19.9)$ \\
& $10-19$ & 30 & 5 & 8 & 5 & $48(9.6)$ \\
& $20-24$ & 24 & 13 & 18 & 13 & $68(13.5)$ \\
& $25-49$ & 102 & 25 & 31 & 65 & $253(50.6)$ \\
& $\geq 50$ & 10 & 12 & 100 & $33(6.4)$ \\
& Total & 200 & 72 & 130 & 502 \\
\hline Sex & Female & 132 & 34 & 62 & 65 & $293(58.4)$ \\
& Male & 68 & 38 & 38 & 65 & $209(41.6)$ \\
\hline Pregnancy status & Yes & 29 & 0 & 0 & 0 & 29 \\
& No & 103 & 32 & 54 & 0 & 189 \\
\hline Lactating status & Yes & 2 & 0 & 2 & 0 & 4 \\
& No & 129 & 32 & 52 & 0 & 213 \\
\hline
\end{tabular}

Abbreviations: HFSUH, Hiwot Fana Specialized University Hospital; FHPH, Federal Harar Police Hospital; JH, Jugel Hospital; SECIIIH, Southeast Command III Hospital.

Table 2 Wards and OPDs Where Patients Treated with Amoxicillin in Selected Governmental Hospitals of Eastern Ethiopia in 2016 ( $=502)$

\begin{tabular}{|c|c|c|c|c|c|}
\hline Departments & JH & FHPH & HFSUH & SECIIIH & Overall Freq (\%) \\
\hline Medical OPD & 69 & 29 & 43 & 118 & $259(5 \mid .7)$ \\
\hline Pediatric OPD & 32 & 14 & 31 & 12 & $89(17.8)$ \\
\hline Emergency OPD & 39 & 29 & 9 & 0 & 77 (15.4) \\
\hline Gyn/obs OPD & 56 & 0 & 2 & 0 & $58(11.6)$ \\
\hline Medical Ward & 1 & 0 & 7 & 0 & $8(1.6)$ \\
\hline Pediatric Ward & 0 & 0 & 5 & 0 & $5(1.0)$ \\
\hline Surgery & 2 & 0 & 1 & 0 & $3(0.6)$ \\
\hline TB Clinic & - & - & - & 2 & $2(0.4)$ \\
\hline ART Clinic & 1 & - & - & - & $\mathrm{I}(0.2)$ \\
\hline Total & 200 & 72 & 100 & 130 & 502 \\
\hline
\end{tabular}

Abbreviations: HFSUH, Hiwot Fana Specialized University Hospital; FHPH, Federal Harar Police Hospital; JH, Jugel Hospital; SECIIIH, Southeast Command III Hospital; OPD, outpatient department; Gyn/obs, gynecology and obstetrics; TB, tuberculosis; ART, antiretroviral therapy.

the most frequent laboratory investigation performed during treatment (Table 3). There was no discharge summary in the vast majority of patients treated with amoxicillin. Duration of hospital stay was less than one day in most cases. The treatment outcome was not documented in the majority of medical records (Table 4).

\section{Amoxicillin Utilization Pattern}

The top amoxicillin clinical indications include nonspecific upper respiratory tract infections (URTIs) $(n=76$, $15.1 \%)$ followed by pneumonia $(n=68,13.5 \%)$ and dental problems $(n=53,10.6 \%$ ) (Table 5). Paracetamol and nonsteroidal anti-inflammatory drugs (NSAIDs), including diclofenac, tramadol, and ibuprofen accounted for $56.2 \%$ (340) of the co-administered medications with amoxicillin therapy followed by antimicrobial agents such as metronidazole, ceftriaxone, and azithromycin which accounted for $29.9 \%$ (181) (Table 6).

\section{Drug Use Evaluation as per Ethiopian STG}

According to Ethiopian STG drug use evaluation criteria, amoxicillin therapy was found appropriate in nearly onefourth of patients treated in these hospitals $(n=120,24 \%)$ (Figure 1). Among the components of the evaluation criteria, the wrong indication was identified as a predominant reason for the inappropriate utilization of this drug $(n=$ 219, 65.4\%) (Figure 2).

\section{Discussion}

In this study, amoxicillin was used in all age groups including pregnant and lactating women. The majority 
Table 3 Laboratory Tests Performed During Amoxicillin Therapy in Selected Governmental Hospitals of Eastern Ethiopia in 2016 $(n=502)$

\begin{tabular}{|l|l|l|l|l|l|}
\hline Laboratory tests & JH & FHPH & HFSUH & SECIIIH & Overall Frequency (\%) \\
\hline CBC & 79 & 5 & 29 & 12 & $125(24.90)$ \\
Urine analysis & 2 & 2 & 2 & 8 & $14(2.78)$ \\
LFT & 2 & - & - & - & $2(0.4)$ \\
Chemistry & 1 & 1 & 7 & - & $9(1.8)$ \\
Stool examination & 2 & 2 & & - & $4(0.8)$ \\
Serology & - & 2 & 1 & - & $3(0.6)$ \\
Blood film & - & - & - & - & - \\
$\quad$ Total & 86 & 12 & 39 & 20 & 157 \\
\hline
\end{tabular}

Abbreviations: HFSUH, Hiwot Fana Specialized University Hospital; FHPH, Federal Harar Police Hospital; JH, Jugel Hospital; SECIIIH, Southeast Command III Hospital; CBC, complete blood count; LFT, liver function test.

Table 4 Outcome of Amoxicillin Therapy in Selected Governmental Hospitals of Eastern Ethiopia in 2016 ( $n=502)$

\begin{tabular}{|c|c|c|c|c|c|c|}
\hline & & JH & FHPH & HFSUH & SECIIIH & Overall Frequency (\%) \\
\hline Outcome of therapy & $\begin{array}{l}\text { Cured } \\
\text { Improved } \\
\text { Not known }\end{array}$ & $\begin{array}{l}1 \\
3 \\
196\end{array}$ & $\begin{array}{l}- \\
- \\
72\end{array}$ & $\begin{array}{l}- \\
8 \\
92\end{array}$ & $\begin{array}{l}- \\
- \\
130\end{array}$ & $\begin{array}{l}\text { I }(0.2) \\
\text { II }(2.2) \\
490(97.6)\end{array}$ \\
\hline Total & & 200 & 72 & 100 & 130 & 502 \\
\hline Discharge summary statement & $\begin{array}{l}\text { Yes } \\
\text { No }\end{array}$ & $\begin{array}{l}185 \\
15\end{array}$ & $\begin{array}{l}3 \\
69\end{array}$ & $\begin{array}{l}22 \\
78\end{array}$ & $\begin{array}{l}- \\
130\end{array}$ & $\begin{array}{l}210(41.8) \\
292(58.2)\end{array}$ \\
\hline Total & & 200 & 72 & 100 & 130 & 502 \\
\hline Duration of stay at hospital (day) & $\begin{array}{l}\leq 1 \\
2-5 \\
>5 \\
\text { Not known }\end{array}$ & $\begin{array}{l}144 \\
4 \mid \\
6 \\
9\end{array}$ & $\begin{array}{l}- \\
- \\
- \\
72\end{array}$ & $\begin{array}{l}25 \\
1 \\
4 \\
70\end{array}$ & $\begin{array}{l}- \\
- \\
- \\
130\end{array}$ & $\begin{array}{l}169(33.7) \\
42(8.4) \\
10(1.2) \\
281(56)\end{array}$ \\
\hline Total & & 200 & 72 & 100 & 130 & 502 \\
\hline
\end{tabular}

Abbreviations: HFSUH, Hiwot Fana Specialized University Hospital; FHPH, Federal Harar Police Hospital; JH, Jugel Hospital; SECIIIH, Southeast Command III Hospital.

(96.2\%) of patients were treated in the outpatient departments. Complete blood count was the most frequent laboratory investigation found in $24.9 \%$ of patient records whereas microbiological culture was not found in any of the records. Top three indications for prescribing amoxicillin were nonspecific upper respiratory tract infections $(15.1 \%)$, pneumonia $(13.5 \%)$, and dental problems (10.6\%). Non-steroidal anti-inflammatory drugs $(56.2 \%)$ were the most frequently co-administered agents. An appropriate utilization was made considering indication, dose, frequency, and duration of therapy in $23.9 \%$ as per the Ethiopian STG. The primary reason found for inappropriateness was the wrong indication $(65.4 \%)$, followed by dose (14.6\%), and duration of therapy (12.2\%).

The administration of antimicrobials primarily depends on the Vivo bioavailability of the drug, in addition to the susceptibility of the bacteria. Other factors also influence the choice of antimicrobial medication, such as the age (infants, children), pregnancy/lactation, and the general state of the patient. ${ }^{48}$ Hence, the common use of amoxicillin in this study might be attributed to its safety and spectrum of activity. It is a pregnancy category B approved drug by the US Food and Drug Administration (FDA) and is considered safe to be taken in any trimester of pregnancy. Clinical evaluations also revealed no association between exposure to the drug and incidence of major birth defects, miscarriage, or adverse maternal or fetal outcomes. ${ }^{49-53}$ In addition, data from literature explained the presence of amoxicillin in breast milk is low with approximately $1 \%$ of the administered dose reaching the infants. A drug with a relative infant dose less than $10 \%$ is considered to be compatible with breastfeeding ${ }^{54-56}$ that may further explain why high prescribing patterns of this drug in all populations. ${ }^{57,58}$ 
Table 5 Top Amoxicillin Indications in Selected Governmental Hospitals of Eastern Ethiopia in $2016(n=502)$

\begin{tabular}{|l|l|l|l|l|l|}
\hline Indications & JH & FHPH & HFSUH & SECIIIH & Overall Frequency (\%) \\
\hline URTIs & 13 & 23 & 7 & 33 & $76(15.1)$ \\
Pneumonia & 14 & 1 & 42 & 1 I & $68(13.5)$ \\
Multiple Indications & 27 & 11 & 7 & 9 & $54(10.8)$ \\
Dental problem & 33 & 2 & 1 & 17 & $53(10.6)$ \\
During/Post Delivery & 47 & 0 & 2 & 0 & $49(9.8)$ \\
Tonsillitis & 11 & 4 & 3 & 16 & $34(6.8)$ \\
Injury & 22 & 2 & 1 & 15 & $29(5.8)$ \\
Bronchitis & 0 & 3 & 4 & 0 & $22(4.4)$ \\
UTI & 6 & 5 & 0 & $15(3)$ \\
Tonsillo pharyngitis & 8 & 6 & 11 & $14(2.8)$ \\
Common cold & 0 & 0 & 0 & $11(2.2)$ \\
Conjunctivitis & 0 & 0 & 0 & 0 & $10(2)$ \\
AFI & 3 & 10 & 3 & 0 & $8(1.6)$ \\
Otitis media & 4 & 2 & 2 & 1 & $7(1.4)$ \\
PUD/Dyspepsia & 0 & 1 & 4 & $6(1.2)$ \\
Asthma & 0 & 1 & 0 & 0 & $4(0.8)$ \\
AGE & 0 & 0 & 2 & 9 & $3(0.6)$ \\
Others & 12 & 1 & 11 & 130 & $39(7.8)$ \\
Total & 200 & 72 & 100 & 502 \\
\hline
\end{tabular}

Abbreviations: HFSUH, Hiwot Fana Specialized University Hospital; FHPH, Federal Harar Police Hospital; JH, Jugel Hospital; SECIIIH, Southeast Command III Hospital; URTIs, upper respiratory tract infections; UTI, urinary tract infection; AFI, acute febrile illness; PUD, peptic ulcer disease; AGE, acute gastroenteritis.

Table 6 Top Co-Administered Drugs with Amoxicillin in Selected Governmental Hospitals of Eastern Ethiopia in 2016 ( $\mathrm{n}=502$ )

\begin{tabular}{|l|l|l|l|l|l|}
\hline Drugs & JH & FHPH & HFSUH & SECIIIH & Overall Frequency (\%) \\
\hline Diclofenac & 66 & 22 & 13 & 55 & $156(25.8)$ \\
Paracetamol & 38 & 25 & 14 & 26 & $103(17.0)$ \\
Metronidazole & 50 & 2 & 16 & 8 & $76(12.6)$ \\
Tramadol & 54 & 5 & 1 & - & $60(9.9)$ \\
Ceftriaxone & 33 & 2 & 2 & $52(6.9)$ \\
Omeprazole & 7 & 5 & 8 & 9 & $29(4.8)$ \\
Azithromycin & 10 & - & 12 & - & $22(3.6)$ \\
Cough syrup & 2 & - & 19 & $22(3.6)$ \\
Ibuprofen & 12 & 4 & - & 17 & $21(3.5)$ \\
Almetamine & - & - & - & $19(3.1)$ \\
Ciprofloxacin & 1 & 3 & - & $17(2.8)$ \\
Chloramphenicol & 1 & - & - & $11(1.8)$ \\
Ampicillin & 8 & 13 & - & $9(1.5)$ \\
Pitocin & 9 & 10 & - & $9(1.5)$ \\
Cimetidine & 1 & 1 & - & $5(0.8)$ \\
Doxycycline & 2 & - & & $4(0.7)$ \\
\hline Total & 2 & 1 & 605 \\
\hline
\end{tabular}

Abbreviations: HFSUH, Hiwot Fana Specialized University Hospital; FHPH, Federal Harar Police Hospital; JH, Jugel Hospital; SECIIIH, Southeast Command III Hospital.

Another finding was the majority $(n=483,96.2 \%)$ of patients treated with amoxicillin were from the outpatient departments (OPDs), with medical OPD the most frequent. Also, few patients $(n=52,10.4 \%)$ had a hospital stay greater than a day. These findings are likely the reasons for the unknown outcome of therapy in most patients, indicating less chance of revisiting the health facilities for follow-up of most ailments.

Considering laboratory services, routine but nonspecific investigations ( $\mathrm{CBC}$, urine analysis, and blood chemistry) were executed for a portion of patients treated with amoxicillin. However, there was no evidence that microbiological 


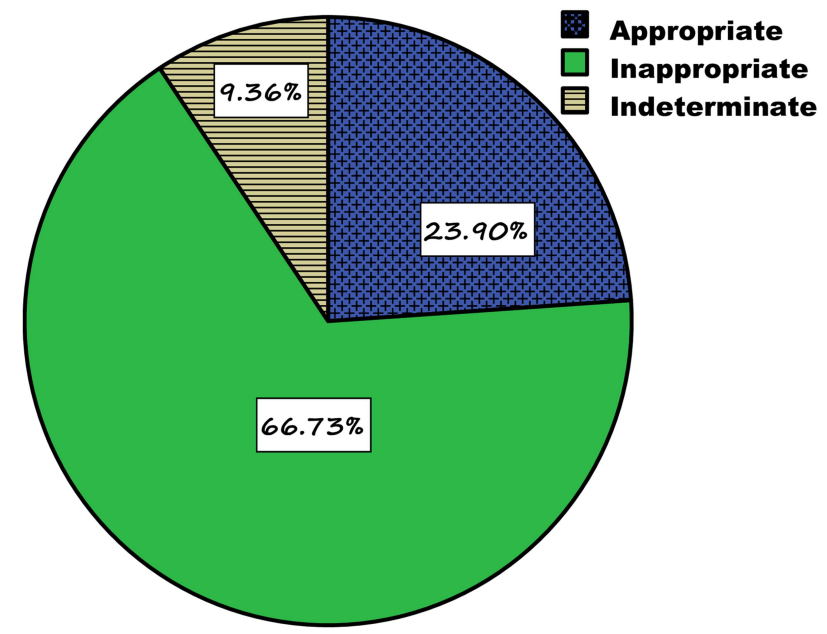

Figure I Appropriateness of amoxicillin indication in selected governmental hospitals (JH, HFSUH, FHPH, and SECIIIH) of Eastern Ethiopia in 2016.

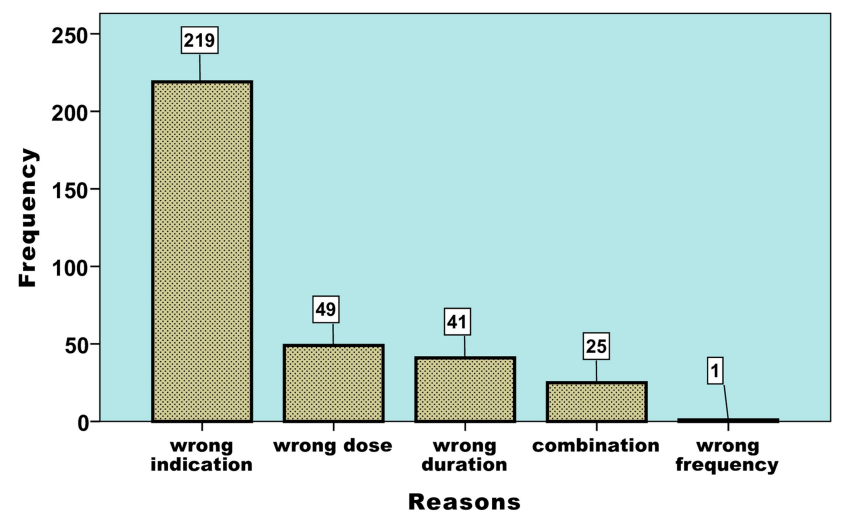

Figure 2 Reasons for inappropriateness in selected governmental hospitals $\mathrm{JH}$, HFSUH, FHPH, and SECIIIH) of Eastern Ethiopia in 2016.

culture and antibiogram tests were performed prior to the administration of the antimicrobial illustrating that antibiotics are being used empirically. It is clear that empirical therapy erodes the effectiveness of available antimicrobial agents which were previously effective and potentially leading to a post-antibiotic era. World Health Organization (WHO) recommends rapid microbial testing and definitive therapy to slow down the antimicrobial resistance. ${ }^{59}$ The analysis of the pharmacokinetic nature of penicillin including amoxicillin shows that it is primarily eliminated unchanged in the urine, ${ }^{60}$ yet simple urinalysis and renal function tests were found absent in $>80 \%$ of cases. Similar practices of empirical therapy were reported in research conducted in public primary healthcare facilities in Addis Ababa, Ethiopia, ${ }^{22}$ Bahir Dar city administration, Northwest Ethiopia, ${ }^{27}$ and from a systemic review on antibiotic use evaluation in India. ${ }^{45}$
Regarding conditions for which amoxicillin was prescribed, non-specific URTIs ( $\mathrm{n}=76,15.1 \%)$ was the most frequent indication. A similar primary indication for URTIs was reported in other towns of Ethiopia health facilities such as Addis Ababa, ${ }^{22}$ Gondar, ${ }^{61}$ Hawassa, ${ }^{26}$ Bahir Dar, ${ }^{35}$ and Dessie. ${ }^{36}$ Moreover, the result from the Euro-Mediterranean region, ${ }^{62}$ Malaysia, $^{21}$ and Yemen ${ }^{63}$ presented an even greater utilization pattern of amoxicillin for nonspecific URTI.

Clinicians speculated most URT infections are of viral origin in nature, though bacteria can cause such infections in some instances. ${ }^{64}$ Therefore, prescribing this drug for non-specific diseases without evidence of pathogenic organism further increases the perception by patients that amoxicillin treatment is the appropriate medication for all URT symptoms, increasing inappropriate self-medication practices. As verified by literature, the most common reasons to practice self-medication were the previous experience of clients and/or familiarity with treatments, ${ }^{65,66}$ and among the most common group of diseases in which patients self-medicated were respiratory symptoms. ${ }^{27,67-69}$ Moreover, among all the antibiotics misused in this practice, penicillin was ranked highest and amoxicillin was most frequently used in this group. ${ }^{66,70}$

Based on the Ethiopian STG, an indication for amoxicillin should specify in terms of dose, frequency, and duration considering previous patient history and severity of the disease. Accordingly, in this research, appropriateness was defined based on the four pertinent parameters, ie, indication, dose, frequency, and duration. An appropriate amoxicillin utilization was found in 120 (23.9\%) of patients. While analyzing reasons for inappropriateness, the wrong indication was the leading practice observed in nearly two-thirds of patients treated with amoxicillin $(\mathrm{n}=219,65.4 \%)$. Indication for non-specific URTI, common cold, asthma, delivery, and accident/injury were some of the wrong indications identified.

Although there was no study reported on the evaluation of amoxicillin utilization to compare, antibacterial with the same mechanism of action, ie, inhibition of cell wall synthesis was considered. Therefore, a research report from the pediatric ward of Jimma University Specialized Hospital indicated the crystalline penicillin utilization adhered to the STGs of Ethiopia and WHO guidelines for the majority of the parameters, while the inappropriateness was primarily exhibited with dose and duration of therapy. ${ }^{71}$ Similar inappropriate utilization was reported 
with a vancomycin therapy study in $\operatorname{Iran}^{9}$ and thirdgeneration cephalosporin utilization in India. ${ }^{72}$

It is interesting to note that maximum levels of antibiotics are used in the community and primary care setting, inappropriately for mostly self-limiting infections. ${ }^{45}$ Health authorities have strongly encouraged physicians to decrease the prescribing of antibiotics to treat common URTIs since antibiotic usage does not significantly reduce recovery time for these illnesses which are often viral. ${ }^{73}$ Some have advocated a delayed antibiotic approach to treating URTIs, which seeks to reduce the consumption of antibiotics while attempting to maintain patient satisfaction. $^{74}$

In addition to non-specific indications, wrong dosage and duration were the causes for inappropriateness which is concerning because dose and duration are critical for penicillin-like amoxicillin since it is a time-dependent bactericidal antibiotic; once the concentration of the drug exceeds the minimum inhibitory concentration (MIC) of the organism, time of exposure (duration) is important for the effectiveness of the drug and since MICs for different bacterial species varies, the dose is dependent on the different bacterial pathogens. ${ }^{75,76}$ Therefore, effectiveness is threatened by antimicrobial resistance that can arise from suboptimal dosing and discontinuation of the full course of treatment. Another important finding was the high usage of NSAIDs which could explain infections related to inflammation, pain, and pyrexia which were treated by such agents. ${ }^{77}$

Although different selection criteria and ways of antimicrobial utilization vary in different parts of the world, amoxicillin is still classified as one of the critically important antibiotics. ${ }^{59,78,79}$ Hence, excessive and inappropriate use is potentially associated with the development of resistance as it was reported from countries with the highest rate of consumption. ${ }^{41}$ Likewise, an increase in the difficulty to treat pathogens has made the selection of appropriate antibiotic therapy a real challenge for presentday clinicians. ${ }^{80}$ Hence, it is worthwhile to implement a standard use of available agents to preserve the antimicrobials and contain AMR.

\section{Conclusion and Recommendation}

Amoxicillin was used in all age groups and populations including pregnant and nursing mothers. The majority of patients were treated in the medical OPD. Laboratory investigations were performed in less than one-third of patients without any evidence of microbiological culture and antibiogram before administration of amoxicillin. The most common indications were URTIs and pneumonia. Based on the Ethiopian STGs, appropriate utilization was found in nearly a quarter of patients. The wrong indications which were identified included utilization in nonspecific URTIs, common cold, asthma, delivery, and accident/ injury. In addition, effectiveness may be compromised by AMR that can arise from suboptimal dosing and premature discontinuation of treatment. Besides, NSAIDs and antimicrobials were the most common co-administered agents. Further studies considering microbiological culture and sensitivity tests, as well as factors that contribute to inappropriate utilization need to be conducted in the study area.

\section{Strength and Limitation}

Amoxicillin use evaluation study was the only study conducted by considering patient characteristics in the four government hospitals of Harar town. Hence, it was possible to compare results from various perspectives, and a summary of findings enabled us to display the practice of utilization patterns of the drug in general in the selected town. Poor documentation in the facilities was the main limitation as the study used secondary data. Moreover, since the study is a descriptive cross-sectional, the underlying reasons why this problem exists could not be established at this time.

\section{Data Sharing Statement}

The data used to support the findings of this study are included in the article.

\section{Ethical Considerations}

This study was conducted in accordance with the Declaration of Helsinki. Prior to beginning any data collection, ethical approval was obtained from the Institutional Health Research Ethics Review Committee (IHRERC), College of Health and Medical Sciences, Haramaya University with reference number: IHRERC/ 078/2017. Permission letters were also received from Harari regional health office and respective hospital administrators to conduct this study. Voluntary, informed, written, and signed consent was obtained from the administrator of each hospital. Since the study was conducted from secondary data (medication records), informed consent was not required from the patients or healthcare professionals themselves. Nevertheless, the confidentiality of the prescribers and patient's information was maintained in such a way that the data abstraction format was 
kept anonymous and data obtained from the hospitals was solely used for the study purpose.

\section{Acknowledgments}

Authors thank data collectors and staff of all hospitals; without them, this research would not be realized.

\section{Author Contributions}

All authors made a significant contribution to the work reported, starting from its conception, study design, execution, and acquisition of data, analysis, and interpretation. Besides, all took part in drafting, revising, or critically reviewing the article; gave final approval of the version to be published; have agreed on the journal to which the article has been submitted; and agree to be accountable for all aspects of the work.

\section{Funding}

The authors disclosed receiving financial support from Haramaya University for conducting this research work.

\section{Disclosure}

The authors have declared that there is no competing interest.

\section{References}

1. Ferech M, Coenen S, Malhotra-Kumar S, et al. European surveillance of antimicrobial consumption (ESAC): outpatient antibiotic use in Europe. J Antimicrob Chemother. 2006;58(2):401-407.

2. van Bijnen EM, den Heijer CD, Paget WJ, et al. The appropriateness of prescribing antibiotics in the community in Europe: study design. BMC Infect Dis. 2011;11(1):293. doi:10.1186/1471-2334-11-293

3. Ahiabu M-A, Tersbøl BP, Biritwum R, Bygbjerg IC, Magnussen P. A retrospective audit of antibiotic prescriptions in primary health-care facilities in Eastern Region, Ghana. Health Policy Plan. 2015;31 (2):250-258. doi:10.1093/heapol/czv048

4. Buke AC, Ermertcan S, Hosgor-Limoncu M, Ciceklioglu M, Eren S. Rational antibiotic use and academic staff. Int $J$ Antimicrob Agents. 2003;21(1):63-66. doi:10.1016/S0924-8579(02)00272-8

5. Arda B, Sipahi OR, Yamazhan T, et al. Short-term effect of antibiotic control policy on the usage patterns and cost of antimicrobials, mortality, nosocomial infection rates and antibacterial resistance. $J$ Infect. 2007;55(1):41-48. doi:10.1016/j.jinf.2007.02.014

6. Gajdács M, Albericio F. Antibiotic Resistance: From the Bench to Patients. Multidisciplinary Digital Publishing Institute; 2019.

7. Gajdács M, Paulik E, Szabó A. Knowledge, attitude and practice of community pharmacists regarding antibiotic use and infectious diseases: a cross-sectional survey in Hungary (KAPPhA-HU). Antibiotics. 2020;9(2):41. doi:10.3390/antibiotics9020041

8. Ayinalem GA, Gelaw BK, Belay AZ, Linjesa JL. Drug use evaluation of ceftriaxone in medical ward of Dessie Referral Hospital, North East Ethiopia. Int J Chem Nat Sci. 2013;1(1):12-16.

9. Hamishehkar H, Ebrahimi D, Mahmoodpoor A, Mashayekhi S, Asgharian P, Reazee H. Drug utilization evaluation of vancomycin in a teaching hospital in Tabriz-Iran. Pharm Sci. 2015;21(1):25. doi:10.15171/PS.2015.13
10. Gajdács M. The concept of an ideal antibiotic: implications for drug design. Molecules. 2019;24(5):892. doi:10.3390/molecules24050892

11. Aslam A, Gajdács M, Zin CS, et al. Evidence of the practice of self-medication with antibiotics among the lay public in low-and middle-income countries: a scoping review. Antibiotics. 2020;9 (9):597. doi:10.3390/antibiotics9090597

12. Aslam A, Gajdács M, Zin CS, Binti Abd Rahman NS, Ahmed SI, Jamshed SQ. Public awareness and practices towards self-medication with antibiotics among the Malaysian population. A development of questionnaire and pilot-testing. Antibiotics. 2020;9(2):97. doi:10.3390/antibiotics9020097

13. Williams VR, Callery S, Vearncombe M, Simor AE. The role of colonization pressure in nosocomial transmission of methicillin-resistant Staphylococcus aureus. Am J Infect Control. 2009;37(2):106-110. doi:10.1016/j.ajic.2008.05.007

14. Lipsitch M, Samore MH. Antimicrobial use and antimicrobial resistance: a population perspective. Emerg Infect Dis. 2002;8(4):347. doi:10.3201/eid0804.010312

15. Wise L. Risks and benefits of (pharmaco) epidemiology. Ther Adv Drug Saf. 2011;2(3):95-102. doi:10.1177/2042098611404920

16. Fahimi F, Baniasadi S, Behzadnia N, Varahram F, Ghazi Tabatabaie L. Enoxaparin utilization evaluation: an observational prospective study in medical inpatients. Iran $J$ Pharm Sci. 2010;77-82.

17. Prescott JF. Beta-lactam antibiotics: penam penicillins. Antimicrob Ther Vet Med. 2006;3:105-133.

18. Blumental D, Brunton LL. Goodman \& Gilman's Manual of Pharmacology and Therapeutics. 2008:728-750.

19. Prevention ECfD, Control. Antimicrobial Consumption in the EU/ EEA, Annual Epidemiological Report for 2018. Stockholm: ECDC; 2019.

20. Mollahaliloglu S, Alkan A, Donertas B, Ozgulcu S, Akici A. Assessment of antibiotic prescribing at different hospitals and primary health care facilities. Saudi Pharm J. 2013;21(3):281-291. doi:10.1016/j.jsps.2012.10.004

21. Ab Rahman N, Teng CL, Sivasampu S. Antibiotic prescribing in public and private practice: a cross-sectional study in primary care clinics in Malaysia. BMC Infect Dis. 2016;16(1):208. doi:10.1186/ s12879-016-1530-2

22. Worku F, Tewahido D. Retrospective assessment of antibiotics prescribing at public primary healthcare facilities in Addis Ababa, Ethiopia. Interdiscip Perspect Infect Dis. 2018;2018:2018. doi:10.1155/2018/4323769

23. Adisa R, Fakeye TO, Aindero VO. Evaluation of prescription pattern and patients' opinion on healthcare practices in selected primary healthcare facilities in Ibadan, South-Western Nigeria. Afr Health Sci. 2015;15(4):1318-1329. doi:10.4314/ahs.v15i4.35

24. Dong L, Yan H, Wang D. Antibiotic prescribing patterns in village health clinics across 10 provinces of Western China. J Antimicrob Chemother. 2008;62(2):410-415. doi:10.1093/jac/dkn153

25. Bilal AI, Osman ED, Mulugeta A. Assessment of medicines use pattern using World Health Organization's prescribing, patient care and health facility indicators in selected health facilities in eastern Ethiopia. BMC Health Serv Res. 2016;16(1):144. doi:10.1186/ s12913-016-1414-6

26. Desalegn AA. Assessment of drug use pattern using WHO prescribing indicators at Hawassa University teaching and referral hospital, south Ethiopia: a cross-sectional study. BMC Health Serv Res. 2013;13(1):170. doi:10.1186/1472-6963-13-170

27. Gebeyehu E, Bantie L, Azage M, Ciccozzi M. Inappropriate use of antibiotics and its associated factors among urban and rural communities of Bahir Dar City administration, Northwest Ethiopia. PLoS One. 2015;10(9):e0138179. doi:10.1371/journal.pone.0138179

28. Ocan M, Bwanga F, Bbosa GS, et al. Patterns and predictors of self-medication in northern Uganda. PLoS One. 2014;9(3):e92323. doi:10.1371/journal.pone.0092323 
29. Widayati A. Self Medication with Antibiotics in Yogyakarta City Indonesia. 2013.

30. Ramay BM, Lambour P, Cerón A. Comparing antibiotic self-medication in two socio-economic groups in Guatemala City: a descriptive cross-sectional study. BMC Pharmacol Toxicol. 2015;16 (1):11. doi:10.1186/s40360-015-0011-3

31. Mitsi G, Jelastopulu E, Basiaris H, Skoutelis A, Gogos C. Patterns of antibiotic use among adults and parents in the community: a questionnaire-based survey in a Greek urban population. Int $J$ Antimicrob Agents. 2005;25(5):439-443. doi:10.1016/j. ijantimicag.2005.02.009

32. Skliros E, Merkouris P, Papazafiropoulou A, et al. Self-medication with antibiotics in rural population in Greece: a cross-sectional multicenter study. BMC Fam Pract. 2010;11(1):58. doi:10.1186/14712296-11-58

33. Togoobaatar G, Ikeda N, Ali M, et al. Survey of non-prescribed use of antibiotics for children in an urban community in Mongolia. Bull World Health Organ. 2010;88(12):930-936. doi:10.2471/ BLT.10.079004

34. Organization WH. WHO Report on Surveillance of Antibiotic Consumption: 2016-2018 Early Implementation. 2018.

35. Fenta A, Belay M, Mekonnen E. Assessment of antibacterial drug exposure patterns of patient encounters seen by different categories of prescribers at health institutions in Bahir Dar, Ethiopia. Ethiop Med J. 2013;51(1):33-39.

36. Getachew E, Aragaw S, Adissie W, Agalu A. Antibiotic prescribing pattern in a referral hospital in Ethiopia. Afr J Pharm Pharmacol. 2013;7(38):2657-2661. doi:10.5897/AJPP12.505

37. Spivak ES, Cosgrove SE, Srinivasan A. Measuring appropriate antimicrobial use: attempts at opening the black box. Clin Infect Dis. 2016;63(12):ciw658. doi:10.1093/cid/ciw658

38. Hara GL, Kanj SS, Pagani L, et al. Ten key points for the appropriate use of antibiotics in hospitalised patients: a consensus from the antimicrobial stewardship and resistance working groups of the international society of chemotherapy. Int J Antimicrob Agents. 2016;48 (3):239-246. doi:10.1016/j.ijantimicag.2016.06.015

39. Slama TG, Amin A, Brunton SA, et al. A Clinician's Guide to the Appropriate and Accurate Use of Antibiotics: The Council for Appropriate and Rational Antibiotic Therapy (CARAT) Criteria. Elsevier; 2005.

40. Kotwani A, Holloway K. Access to antibiotics in New Delhi, India: implications for antibiotic policy. J Pharm Policy Pract. 2013;6(1):6. doi:10.1186/2052-3211-6-6

41. Goossens H, Ferech M, Vander Stichele R, Elseviers M, Group EP. Outpatient antibiotic use in Europe and association with resistance: a cross-national database study. Lancet. 2005;365(9459):579-587. doi:10.1016/S0140-6736(05)70799-6

42. Gajdács M, Paulik E, Szabó A. Public knowledge, attitude and practices towards antibiotics and antibiotic resistance: a cross-sectional study in Szeged District, Hungary. Acta Pharm Hung. 2020;90(1):5-14. doi:10.33892/aph.2020.90.5-14

43. Chlabicz M, Chlabicz S, Marcinowicz L, Rogowska-Szadkowska D, Pytel-Krolczuk B, Leszczyńska K. Outpatient use of systemic antibiotics in Poland: 2004-2008. Przegl Epidemiol. 2014;68 (3):435-441.

44. Sisay M, Gashaw T, Amare F, Tesfa T, Baye Y. Hospital-level antibacterial prescribing and its completeness in ethiopia: did it adhere to good prescribing practice? Int J Gen Med. 2020;13:1025. doi:10.2147/IJGM.S280696

45. Haadi A, Abdullah S, Faqruddin M, Khan MN, Khan MA, Omer M. A systemic review on antibiotic use evaluation in paediatrics. World J Pharm Sci. 2014;2:243-246.

46. HRHB. Harari Reginal State Annual Bulletin. 2014.

47. Food M, Administration HC, Ethiopia CAo. Standard Treatment Guidelines for General Hospitals. 2014.
48. Gajdacs M. Intravenous or oral antibiotic therapy: sophie's choice? Gen Intern Med Clin Innov. 2019;4(2):1-2. doi:10.15761/ GIMCI. 1000176

49. Almeida L, Schmauch A, Bergström S. A randomised study on the impact of peroral amoxicillin in women with prelabour rupture of membranes preterm. Gynecol Obstet Invest. 1996;41(2):82-84. doi:10.1159/000292046

50. Lovett SM, Weiss JD, Diogo MJ, Williams PT, Garite TJ. A prospective, double-blind, randomized, controlled clinical trial of ampicillin-sulbactam for preterm premature rupture of membranes in women receiving antenatal corticosteroid therapy. Am J Obstet Gynecol. 1997;176(5):1030-1038. doi:10.1016/S0002-9378(97)70398-3

51. Mercer BM, Miodovnik M, Thurnau GR, et al. Antibiotic therapy for reduction of infant morbidity after preterm premature rupture of the membranes: a randomized controlled trial. JAMA. 1997;278 (12):989-995. doi:10.1001/jama.1997.03550120049032

52. Czeizel AE, Rockenbauer M, Sørensen HT, Olsen J. Augmentin treatment during pregnancy and the prevalence of congenital abnormalities: a population-based case-control teratologic study. Eur J Obstet Gynecol Reprod Biol. 2001;97(2):188-192. doi:10.1016/ S0301-2115(00)00545-5

53. Jepsen P, Skriver MV, Floyd A, Lipworth L, Schønheyder HC, Sørensen HT. A population-based study of maternal use of amoxicillin and pregnancy outcome in Denmark. Br J Clin Pharmacol. 2003;55(2):216-221. doi:10.1046/j.1365-2125.2003.01750.x

54. Ito S, Blajchman A, Stephenson M, Eliopoulos C, Koren G. Prospective follow-up of adverse reactions in breast-fed infants exposed to maternal medication. Am J Obstet Gynecol. 1993;168 (5):1393-1399. doi:10.1016/S0002-9378(11)90771-6

55. Benyamini L, Merlob P, Stahl B, et al. The safety of amoxicillin/ clavulanic acid and cefuroxime during lactation. Ther Drug Monit. 2005;27(4):499-502. doi:10.1097/01.ftd.0000168294.25356.d0

56. Yazdani-Brojeni P, Garcia-Bournissen F, Fujii H, Tanoshima R, Ito S. Relative bioequivalence of amoxicillin dissolved in breast milk. Arch Dis Child. 2014;99(3):258-261. doi:10.1136/archdischild-2013305151

57. Bookstaver PB, Bland CM, Griffin B, Stover KR, Eiland LS, McLaughlin M. A review of antibiotic use in pregnancy. Pharmacotherapy. 2015;35(11):1052-1062. doi:10.1002/phar.1649

58. Kaur SP, Rao R, Nanda S. Amoxicillin: a broad spectrum antibiotic. Int J Pharm Pharm Sci. 2011;3(3):30-37.

59. WHO. Critically Important Antimicrobials for Human Medicine: Ranking of Antimicrobial Agents for Risk Management of Antimicrobial Resistance Due to Non-Human Use. 2017.

60. Brodie D, Griggs J, Cunningham K. Comparative study of cefuroxime axetil suspension and amoxycillin syrup in the treatment of acute otitis media in general practice. $J$ Int Med Res. 1990;18(3):235-239. doi:10.1177/030006059001800309

61. Abula T, Worku A. Self-medication in three towns of North West Ethiopia. Ethiop J Health Dev. 2001;15(1):25-30. doi:10.4314/ejhd. v15i1.9893

62. Scicluna EA, Borg MA, Gür D, et al. Self-medication with antibiotics in the ambulatory care setting within the Euro-Mediterranean region; results from the ARMed project. $J$ Infect Public Health. 2009;2 (4):189-197. doi:10.1016/j.jiph.2009.09.004

63. Alshakka M, Said K, Babakri M, et al. A study on antibiotics prescribing pattern at outpatient department in four hospitals in aden-yemen. J Pharm Pract Community Med. 2016;2(3):88-93. doi:10.5530/jppcm.2016.3.5

64. Rajpurkar P, Irvin J, Zhu K, et al. Chexnet: radiologist-level pneumonia detection on chest $\mathrm{x}$-rays with deep learning. arXiv Preprint arXiv. 2017.

65. Sisay M, Mengistu G, Edessa D. Epidemiology of self-medication in Ethiopia: a systematic review and meta-analysis of observational studies. BMC Pharmacol Toxicol. 2018;19(1):56. doi:10.1186/ s40360-018-0248-8 
66. Al-Azzam SI, Al-Husein BA, Alzoubi F, Masadeh MM, Al-Horani A. Self-medication with antibiotics in Jordanian population. Int J Occup Med Environ Health. 2007;20(4):373. doi:10.2478/ v10001-007-0038-9

67. Ayalew MB. Self-medication practice in Ethiopia: a systematic review. Patient Prefer Adherence. 2017;11:401. doi:10.2147/PPA. S131496

68. Grigoryan L, Haaijer-Ruskamp FM, Burgerhof JG, et al. Selfmedication with antimicrobial drugs in Europe. Emerg Infect Dis. 2006;12(3):452. doi:10.3201/eid1203.050992

69. Nakhaee M, Vatankhah S. Prevalence and cause of self-medication in iran: a systematic review and meta-analysis on health center based studies. J Biochem Tech. 2019;(2):90-105.

70. Mboya EA, Sanga LA, Ngocho JS. Irrational use of antibiotics in the moshi municipality northern Tanzania: a cross sectional study. Pan Afr Med J. 2018;31:31. doi:10.11604/pamj.2018.31.165.15991

71. Siraj J, Ahmed S. Evaluation of crystalline penicillin use in pediatrics ward of Jimma University specialized hospital, South West Ethiopia. Clin Exp Pharmacol. 2013;3(135):2161-1459.1000135.

72. Kaliamoorthy K, Sankaralingam R, Punniyakotti S, Janardhan V, Cheekala UMR. Drug utilization evaluation of third generation cephalosporins using core drug use indicators. Pak J Pharm Sci. 2012;25(2):339-342.

73. Reveiz L, Cardona AF. Antibiotics for acute laryngitis in adults. Cochrane Database Syst Rev. 2015;(5). doi:10.1002/14651858. CD004783.pub5
74. Nice C. Respiratory Tract Infections-Antibiotic Prescribing: Prescribing of Antibiotics for Self-Limiting Respiratory Tract Infections in Adults and Children in Primary Care. 2008.

75. El Garch F, de Jong A, Simjee S, et al. Monitoring of antimicrobial susceptibility of respiratory tract pathogens isolated from diseased cattle and pigs across Europe, 2009-2012: vetPath results. Vet Microbiol. 2016;194:11-22. doi:10.1016/j.vetmic.2016.04.009

76. Yamamoto K, Kijima M, Yoshimura H, Takahashi T. Antimicrobial susceptibilities of Erysipelothrix rhusiopathiae isolated from pigs with swine erysipelas in Japan, 1988-1998. J Vet Med B. 2001;48 (2):115-126. doi:10.1046/j.1439-0450.2001.00427.x

77. Goldman RD, Society CP, Therapy D, Committee HS. Treating cough and cold: guidance for caregivers of children and youth. Paediatr Child Health. 2011;16(9):564-566. doi:10.1093/pch/ 16.9 .564

78. CVM F. Guidance for Industry\# 152. Evaluating the Safety of Antimicrobial New Animal Drugs with Regard to Their Microbiological Effects on Bacteria of Human Health Concern. Rockville, MD: Center for Veterinary Medicine, Food and Drug Administration; 2003.

79. EMA. Answers to the Requests for Scientific Advice on the Impact on Public Health and Animal Health of the Use of Antibiotics in Animals. 2014.

80. Geddes AM, Gould IM. Ampicillin, Amoxicillin and Other Ampicillin-Like Penicillins. Kucers' the Use of Antibiotics. 6th ed. London (UK): Hodder Arnold; 2010:65.
Infection and Drug Resistance

\section{Publish your work in this journal}

Infection and Drug Resistance is an international, peer-reviewed openaccess journal that focuses on the optimal treatment of infection (bacterial, fungal and viral) and the development and institution of preventive strategies to minimize the development and spread of resistance. The journal is specifically concerned with the epidemiology of antibiotic resistance and the mechanisms of resistance development and diffusion in both hospitals and the community. The manuscript management system is completely online and includes a very quick and fair peerreview system, which is all easy to use. Visit http://www.dovepress.com/ testimonials.php to read real quotes from published authors. 